\title{
Etomidate inhibits cell proliferation and induces apoptosis in A549 non-small cell lung cancer cells via downregulating WWP2
}

\author{
DEQIANG LI ${ }^{1}$, JUNLONG ZHANG ${ }^{2}$, LIJUN YIN ${ }^{1}$, ZHEN JIN $^{2}$, XUEJUN CHEN ${ }^{1}$ and XIANGXUE MENG ${ }^{2}$ \\ ${ }^{1}$ Department of Anesthesiology, Tianjin Baodi Hospital, Baodi Clinical College of Tianjin Medical University, Tianjin 301800; \\ ${ }^{2}$ Department of Anesthesiology, The Second People's Hospital of Lianyungang, Lianyungang, Jiangsu 222000, P.R. China
}

Received March 2, 2021; Accepted June 25, 2021

DOI: $10.3892 / \mathrm{etm} .2021 .10689$

\begin{abstract}
Etomidate (ETO) is a commonly used intravenous anesthetic that has been reported to exert a tumor suppressive effect in several types of cancer. The present study aimed to investigate the effect of ETO on cell proliferation and apoptosis in non-small cell lung cancer (NSCLC) cells and elucidate its potential mechanism of action. Therefore, Cell Counting Kit- 8 assay was performed to evaluate the effect of different concentrations of ETO $(0,1,2$ or $3 \mu \mathrm{g} / \mathrm{ml})$ on A549 cell viability. In addition, the possible interaction between ETO and WW domain containing E3 ubiquitin protein ligase 2 (WWP2) was predicted using the STITCH database. Additionally, a stable WWP2-overexpressing A549 cell line was constructed by transfecting A549 cells with the pcDNA3.1-WWP2 plasmid. Cell proliferation and apoptosis were assessed using colony formation and TUNEL assays, respectively. The mRNA and protein expression levels of the apoptosis-related proteins Bcl-2, Bax, caspase 3 and cleaved-caspase 3 were determined by reverse transcription-quantitative PCR and western blotting. In addition, the expression and phosphorylation levels of proliferation-associated genes (PCNA and Ki-67) and proteins in the PI3K/Akt pathway were analyzed by western blotting. The results showed that treatment with ETO attenuated the cell viability and proliferation of A549 cells. ETO also promoted cell apoptosis and decreased the expression of the anti-apoptotic protein Bcl-2, whilst increasing that of pro-apoptotic proteins Bax and cleaved caspase 3 in a dose-dependent manner. Furthermore, ETO was found to negatively regulate the expression of WWP2, such that WWP2 overexpression reversed the potentiating effects of ETO on cell apoptosis. In addition, ETO promoted the expression of PTEN and reduced the phosphorylation levels of the PI3K/AKT pathway-related
\end{abstract}

Correspondence to: Dr Xiangxue Meng, Department of Anesthesiology, The Second People's Hospital of Lianyungang, 161 Xingfu Road, Lianyungang, Jiangsu 222000, P.R. China E-mail: mengxx212@163.com

Key words: etomidate, non-small cell lung cancer, WW domain-containing E3 ubiquitin protein ligase 2, proliferation, apoptosis proteins. These effects aforementioned could also be reversed by WWP2 overexpression. Therefore, data from the present study suggest that ETO can attenuate the progression of NSCLC through by the PI3K/AKT pathway, specifically by targeting WWP2. These findings may provide a novel target for the treatment of NSCLC.

\section{Introduction}

According to the 2019 US Cancer Statistics report (1), although the incidence of lung cancer is lower compared with that of prostate and breast cancer, lung cancer is associated with the highest rate of cancer-related morbidity in the USA. In China, the morbidity and mortality rates of lung cancer are the highest among all types of cancer (2). Non-small cell lung cancer (NSCLC) is a subtype of lung cancer that accounts for $\sim 85 \%$ of all lung cancer cases worldwide, which is also the main cause of lung cancer-related mortality (3). At present, available clinical treatment options for NSCLC primarily includes surgery and radiotherapy, combined with drug chemotherapy (4-6). However, NSCLC is prone to drug resistance, metastasis and recurrence, leading to poor survival rates (7). Therefore, investigating the molecular mechanism underlying the proliferation, migration and invasion of NSCLC cells is crucial for prolonging the survival of patients with NSCLC.

Etomidate (ETO) is a commonly used intravenous anesthetic that maintains good hemodynamic stability during anesthesia (8). It has been reported that ETO exerts an inhibitory role in several types of cancer. For example, it has been demonstrated that ETO could attenuate the proliferation of human adrenocortical cancer cells (9) and enhance the apoptosis of N2a neuroblastoma cells (10). In addition, ETO was found to significantly inhibit the migratory and invasive abilities of NSCLC cells (11). However, the effect of ETO on the apoptosis of NSCLC cells has not been previously reported. Therefore, the present study aimed to explore the effect of ETO on the proliferation and apoptosis of NSCLC cells.

Subsequently, the STITCH database was used to predict the proteins interacting with ETO and to explore the possible relationship between ETO and WW domain containing E3 ubiquitin protein ligase 2 (WWP2) in the WW domain. WWP2 is a member of the C2-WW-HECT family (NEDD4 family) of E3 ubiquitin ligases (E3), which act as acceptors of ubiquitin from E2 enzymes and then transfer ubiquitin to 
a specific lysine residue on the substrate (12). WWP2 has a role in protecting cartilage from osteoarthritis through runt-related transcription factor 2 (Runx2) polyubiquitination and degradation to inhibit Runx2-induced disintegrin and metalloproteinase with thrombospondin motifs 5 (13). WWP2 is a novel cancer-related factor that has been reported to be associated with the occurrence of liver cancer and lung adenocarcinoma (14). A previous study demonstrated that hypoxia-inducible factor-1 $\alpha$ may promote apoptosis and inhibit the invasion of thyroid cancer cells by downregulating the expression of factors, such as WWP2 (15). Another study showed that the expression of WWP2 was notably upregulated in NSCLC tissues, where WWP2 overexpression could effectively promote the proliferation of NSCLC cells (16). Therefore, it was hypothesized that ETO may affect the progression of NSCLC by interacting with WWP2.

The present study aimed to uncover the role of ETO in the proliferation, migration and apoptosis of NSCLC cells and WWP2 expression, which could hopefully provide a theoretical basis for a novel treatment strategy for NSCLC.

\section{Materials and methods}

Cell culture. A549 cells were purchased from the American Type Culture Collection and maintained in RPMI-1640 medium (Thermo Fisher Scientific, Inc.) supplemented with $10 \%$ FBS (Thermo Fisher Scientific, Inc.) in a $5 \% \mathrm{CO}_{2}$ incubator at $37^{\circ} \mathrm{C}$. BESA-2B cells were also purchased from the American Type Culture Collection and maintained in LHC medium (Thermo Fisher Scientific, Inc.) supplemented with $10 \%$ FBS (Thermo Fisher Scientific, Inc.) in a $5 \% \mathrm{CO}_{2}$ incubator at $37^{\circ} \mathrm{C}$. The cells were passaged once every 3 days, whilst only cells in the logarithmic growth phase were used for the subsequent experiments.

Bioinformatics. The STITCH DataBase (version 5.0; http://stitch.embl.de/) is a database that can be used to explore known and predicted interactions between chemicals and proteins (17). Proteins that directly interact with ETO will be selected as putative targets (minimum required interaction score: 0.400 ).

Cell transfection. The WWP2 overexpression vector, pcDNA3.1-WWP2 and empty control vector, pcDNA3.1-NC, were synthesized by Shanghai GeneChem Co., Ltd.. Cells were seeded onto 12 -well plates at a density of $4 \times 10^{5}$ cells/well and cultured for $24 \mathrm{~h}$ at $37^{\circ} \mathrm{C}$. Following incubation, cells were transfected with the aforementioned plasmids $\left(1.5 \mu \mathrm{g}\right.$ per well) using Lipofectamine ${ }^{\circledR} 2000$ (Invitrogen; Thermo Fisher Scientific, Inc.), according to the manufacturer's protocols. Following transfection for $48 \mathrm{~h}$, the transfection efficiency was evaluated by reverse transcription-quantitative PCR (RT-qPCR). After transfection, 1.0, 2.0 and $3.0 \mu \mathrm{g} / \mathrm{ml}$ ETO (cat. no. A28229; Beijing Wokai Biological Technology Co., Ltd.; https://www.bjoka-vip. com) were added and co-incubated for 24,48 and $72 \mathrm{~h}$ at $37^{\circ} \mathrm{C}$ for subsequent experiments.

Cell counting kit-8 (CCK-8) assay. The cell viability was assessed by CCK-8 assay (Sigma-Aldrich; Merck KGaA).
Briefly, cells were seeded onto 96 -well plates at a density of $2 \times 10^{3}$ cells/well and incubated for 24,48 and $72 \mathrm{~h}$ at $37^{\circ} \mathrm{C}$. Following incubation, $10 \mu \mathrm{l}$ CCK- 8 solution was added into each well and cells were cultured for an additional $2 \mathrm{~h}$ at $37^{\circ} \mathrm{C}$. The absorbance in each well was measured at a wavelength of $450 \mathrm{~nm}$ using a microplate reader (Synergy 2 Multi-Mode Microplate Reader; BioTek Instruments, Inc.).

Colony formation assay. The cells with $4 \times 10^{2}$ cells/well suspended in RPMI-1640 medium were seeded into six-well plates and cultured in a $5 \% \mathrm{CO}_{2}$ incubator at $37^{\circ}$ for 14 days. Subsequently, the cells were fixed with $70 \%$ ethanol at room temperature for $15 \mathrm{~min}$ and stained with $0.05 \%$ crystal violet for $20 \mathrm{~min}$ at $37^{\circ} \mathrm{C}$. The number of colonies formed (>50 cells/colony) were counted under a Olympus BX40 light microscope (magnification, x200; Olympus Corporation).

TUNEL assay. Apoptosis was assessed using the TUNEL Apoptosis Assay Kit (cat. no. C1088; Beyotime Institute of Biotechnology). Briefly, the cells $\left(1 \times 10^{6}\right.$ cells/well) were washed with PBS, fixed at room temperature with $4 \%$ paraformaldehyde for $20 \mathrm{~min}$ and then treated with $0.1 \%$ Triton X-100 for $10 \mathrm{~min}$. Subsequently, $50 \mu 1$ TUNEL detection solution was added to each well, incubated at $37^{\circ} \mathrm{C}$ for $60 \mathrm{~min}$ in dark and washed with PBS three times. A small amount of DAPI staining solution (final concentration: $5 \mathrm{mg} / \mathrm{ml}$ ) was added (covering the sample) and placed at room temperature for 3-5 min and then washed with PBS three times. Anti-fluorescence quenching mounting solution was used to mount the slides (Beyotime Institute of Biotechnology). The morphological changes of apoptotic cells were observed under the AMG EVOS fluorescence microscope (magnification, x200; Thermo Fisher Scientific, Inc.). Three fields of each sample were randomly selected for apoptosis analysis. Cells with green fluorescence were considered to be apoptotic and quantified using the following formula: Cell apoptosis $(\%)=$ Green fluorescence area/total area $\mathrm{x} 100 \%$.

$R T$ - $q$ PCR analysis. Total RNA was extracted from A549 cells using a TRIzol ${ }^{\circledR}$ reagent (Thermo Fisher Scientific, Inc.) and was then reverse-transcribed to cDNA using the FastQuant RT kit (cat. no. KR106; Tiangen Biotech Co., Ltd.) according to the manufacturer's protocol. qPCR reactions were performed using the PowerUp ${ }^{\mathrm{TM}} \mathrm{SYBR}^{\mathrm{TM}}$ Green Master Mix (cat. no. A25779; Applied Biosystems; Thermo Fisher Scientific, Inc.) on the ABI 7500 PCR system (Applied Biosystems; Thermo Fisher Scientific, Inc.). The thermocycling conditions used were as follows: Initial denaturation at $94^{\circ} \mathrm{C}$ for $30 \mathrm{sec}$, followed by 22 cycles at $55^{\circ} \mathrm{C}$ for $30 \mathrm{sec}$ and $72^{\circ} \mathrm{C}$ for $30 \mathrm{sec}$. The relative expression levels of target genes were normalized to those of the housekeeping gene GAPDH and calculated by the $2^{-\Delta \Delta \mathrm{Cq}}$ method (18). The sequences of PCR primers were as follows: Proliferating cell nuclear antigen (PCNA) forward, 5'-GGGTGAAGTTTTCCGCCAGT-3' and reverse, 5'-CTG TAGGAGAAAGCGGAGTGG-3'; Ki-67 forward, 5-ATCCTT ACCTCCCAACCTCTGT-3 and reverse, 5'-AACTTCTGG CTCTTCCTGTAGC-3'; WWP2 forward, 5'-CGGTGTAGG CAGAGCTGATG-3' and reverse, 5'-CCACAAGGCAGA AACACCAA-3'; PTEN forward, 5'-CTCCTACTTCCACCT GCTCAC-3' and reverse, 5'-AAGGATCTCCAGGCTCGA 
A

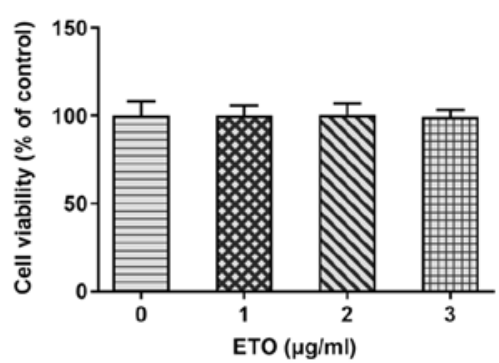

C

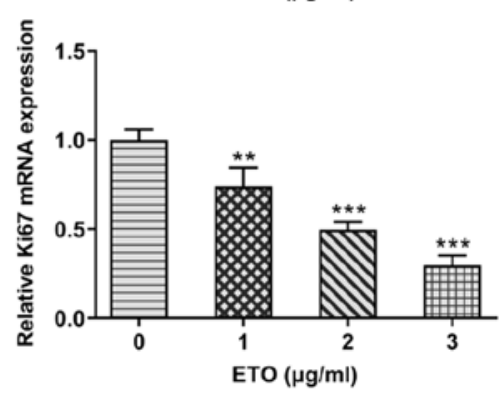

B
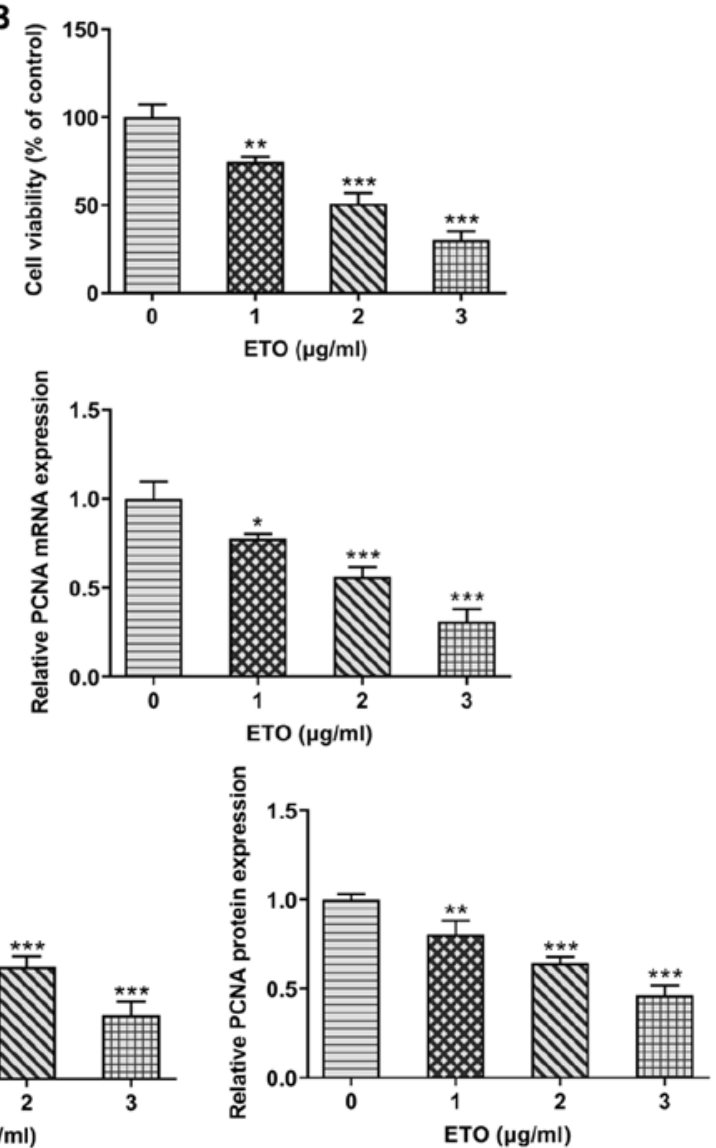

E
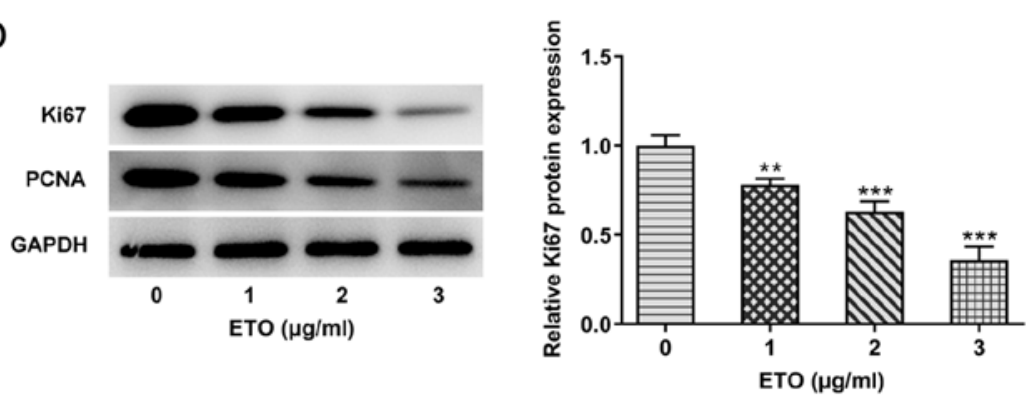

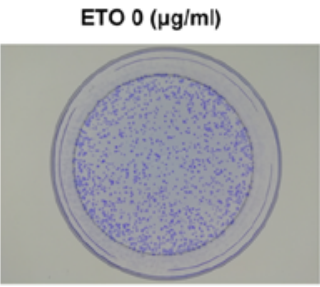

$\operatorname{ETO} 2(\mu \mathrm{g} / \mathrm{ml})$

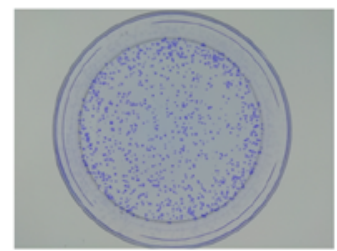

$\operatorname{ETO} 1(\mu \mathrm{g} / \mathrm{ml})$

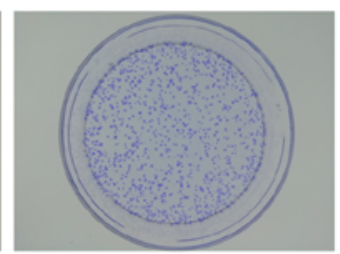

$\operatorname{ETO} 3(\mu \mathrm{g} / \mathrm{ml})$

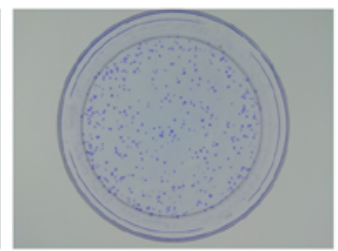

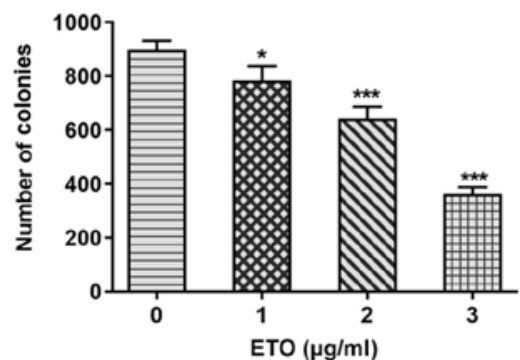

Figure 1.ETO attenuates A549 cell proliferation. (A) BESA-2B cells were treated with different concentrations of ETO $(0,1,2$ or $3 \mu \mathrm{g} / \mathrm{ml})$ for $24 \mathrm{~h}$, before cell viability was measured by Cell Counting Kit-8 assay. (B) A549 cells were treated with different concentrations of ETO ( $0,1,2$ or $3 \mu \mathrm{g} / \mathrm{ml})$ for $24 \mathrm{~h}$ before cell viability was measured the Cell Counting Kit-8 assay. (C) mRNA and (D) protein expression levels of Ki67 and PCNA in A549 cells were detected by reverse transcription-quantitative PCR and western blotting assays, respectively. (E) Cell proliferation was assessed using colony formation assay. ${ }^{*} \mathrm{P}<0.05{ }^{, * *} \mathrm{P}<0.01$ and ${ }^{* * *} \mathrm{P}<0.001 \mathrm{vs.} 0 \mu \mathrm{g} / \mathrm{ml}$ ETO. ETO, etomidate; PCNA, proliferating cell nuclear antigen.

AA-3' and GAPDH forward, 5'-GATGATGTTGAACTCGTC GC-3' and reverse, 5'-CTCTTCTGGGTTTCTCACACC-3'.

Western blot analysis. Total proteins were extracted from A549 cells using the RIPA buffer (cat. no. P0013B; Beyotime Institute of Biotechnology). The protein concentration was measured utilizing a BCA protein quantitative kit (cat. no. P0012; Beyotime Institute of Biotechnology). Subsequently, $20 \mu \mathrm{g}$ protein extracts were separated by $10 \%$ SDS-PAGE and transferred onto PVDF membranes (Beyotime Institute of
Biotechnology). Following blocking with 5\% skimmed milk for $30 \mathrm{~min}$ at room temperature, the membranes were incubated with primary antibodies (dilution, 1:1,000; all from Abcam) against PCNA (cat. no. ab92552), Ki67 (cat. no. ab15580), Bcl-2 (cat. no. ab182858), Bax (cat. no. ab182733), caspase 3 (cat. no. ab32150), cleaved caspase 3 (cat. no. ab2302), WWP2 (cat. no. ab103527), PTEN (cat. no. ab267787), PI3K (cat. no. ab32089), AKT (cat. no. ab18785), phosphorylated (p)-AKT (cat. no. ab38449) and GAPDH (cat. no. ab9485) overnight at $4^{\circ} \mathrm{C}$. The next day, the membranes were incubated with the 
A
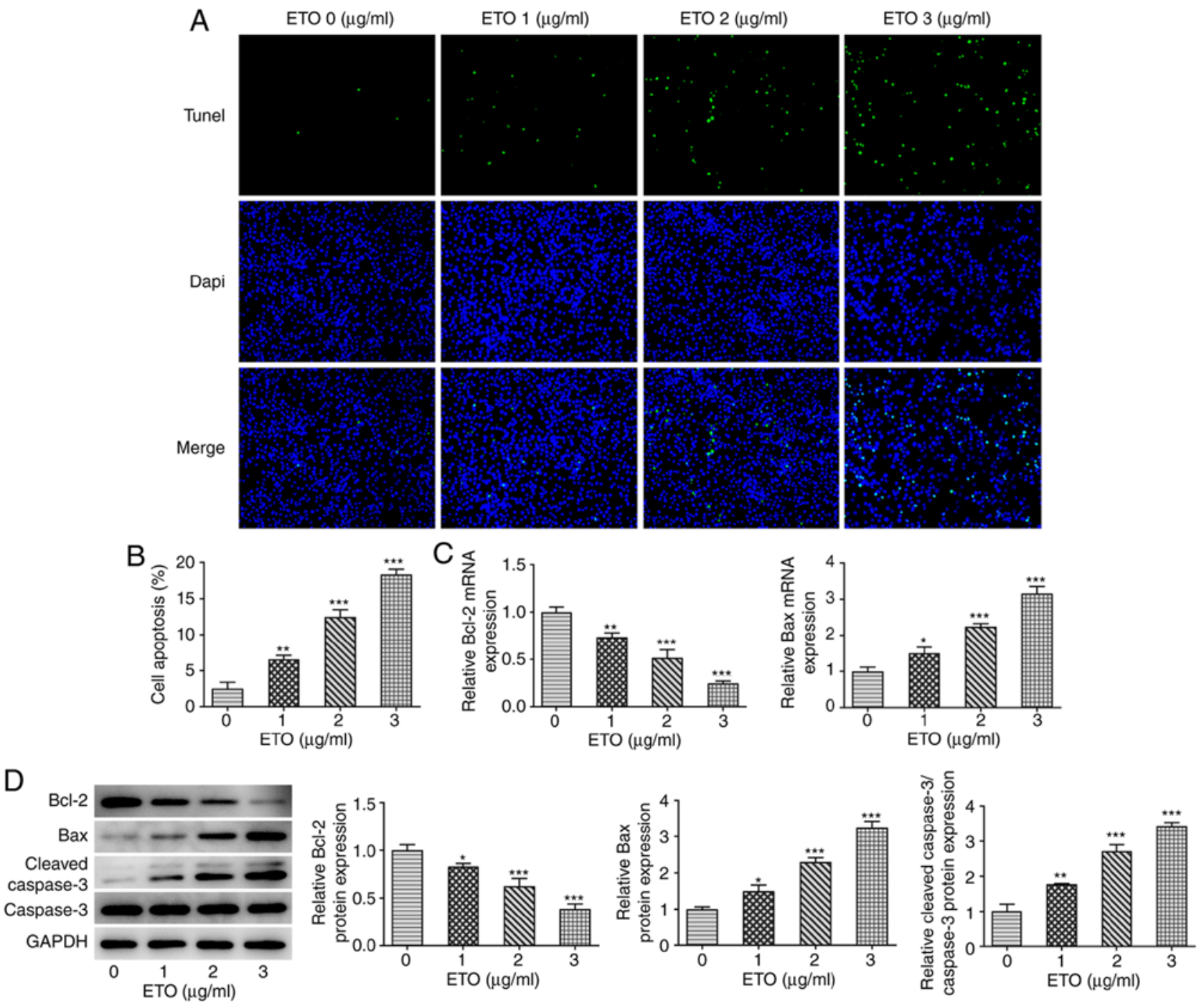

Figure 2. ETO induces apoptosis in A549 cells. (A) A549 cells were treated with different concentrations of ETO (0, 1,2 or $3 \mu$ g/ml) for 24 h, before cell apoptosis was evaluated by TUNEL assay (magnification, x200), (B) the results of which were quantified. (C) mRNA expression levels of Bcl-2 and Bax were determined by reverse transcription-quantitative PCR. (D) Protein expression levels of Bcl-2, Bax, cleaved caspase 3 and caspase 3 were detected by western blot analysis. ${ }^{*} \mathrm{P}<0.05,{ }^{* *} \mathrm{P}<0.01$ and ${ }^{* * * *} \mathrm{P}<0.001$ vs. $0 \mu \mathrm{g} / \mathrm{ml}$ ETO group. ETO, etomidate.

corresponding HRP-conjugated secondary antibodies (cat. no. ab97190; dilution, 1:1,000; Abcam) at $37^{\circ} \mathrm{C}$ for $2 \mathrm{~h}$. The ECL Plus kit (cat. no. P0018; Beyotime Institute of Biotechnology) was utilized to visualize the protein bands (Image $\mathrm{J}$; version number: 1.4.3.67; National Institutes of Health).

Statistical analysis. All data were analyzed with the GraphPad Prism 7 software (GraphPad Software, Inc.). All data are expressed as the mean \pm SD $(n=3)$. Differences between two groups were compared using an unpaired Student's t-test, whilst those among multiple groups by one-way ANOVA followed by Tukey's post hoc test. $\mathrm{P}<0.05$ was considered to indicate a statistically significant difference.

\section{Results}

ETO attenuates A549 cell proliferation and induces apoptosis. First, CCK-8, colony formation and TUNEL assays were performed to evaluate the viability, proliferation and apoptosis of A549 cells, respectively, following treatment with different concentrations of ETO $(0,1,2$ or $3 \mu \mathrm{g} / \mathrm{ml})$. The effect of different concentrations of ETO on the viability of normal lung epithelial BESA-2B cells was tested first, which yielded no difference (Fig. 1A). As shown in Fig. 1B, CCK-8 assay results showed that ETO significantly reduced the viability of A549 cells in a dose-dependent manner. In addition, the inhibitory effects of ETO on the expression of the proliferation-related genes, Ki67 and PCNA (19) was stronger with increasing concentrations of ETO (Fig. 1C and D). The results of colony formation assays showed that ETO also significantly decreased the number of colonies formed in a dose-dependent manner (Fig. 1E).

Subsequently, results from TUNEL assay revealed that, compared with that in the control group, ETO significantly promoted the apoptosis of A549 cells in a dose-dependent manner (Fig. 2A and B). Similarity, the RT-qPCR and western blot analyzes showed that ETO significantly reduced the expression of the anti-apoptotic protein Bcl-2, and increased that of Bax and cleaved caspase 3 in a dose-dependent manner (Fig. 2C and D). 
A

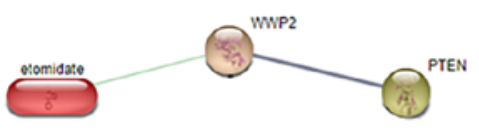

D

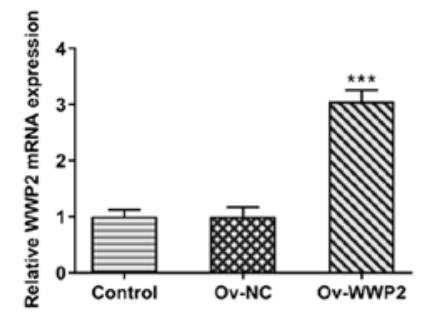

$\mathbf{F}$

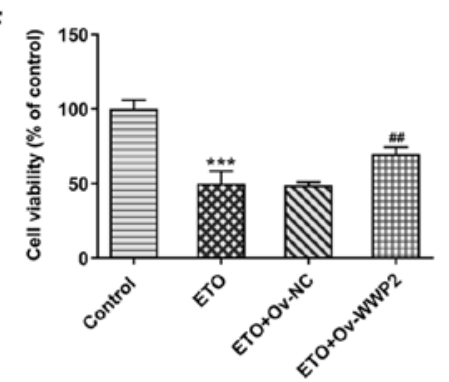

H

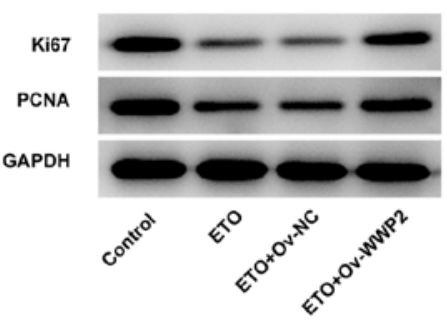

B

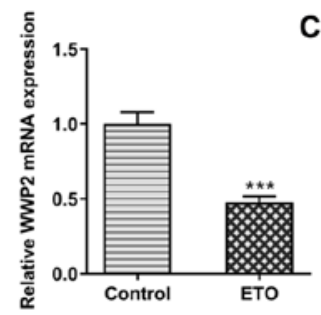

$\mathbf{E}$

WWP2

GAPDH

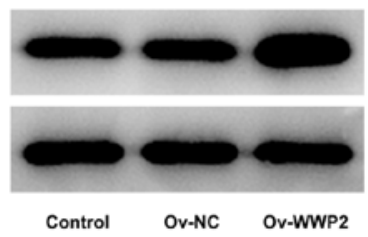

G
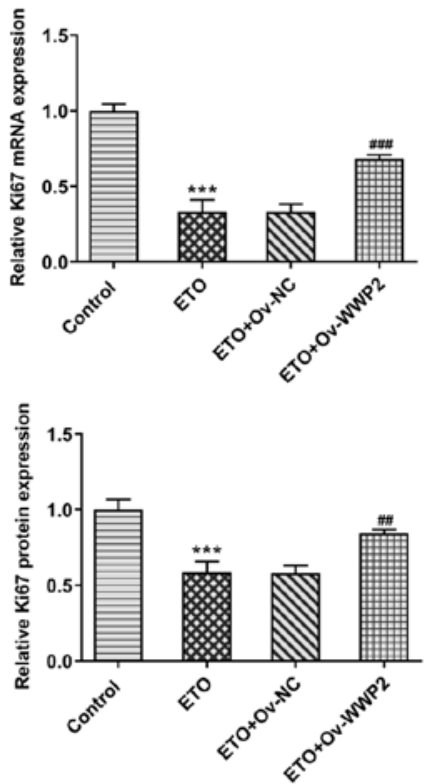

ETO
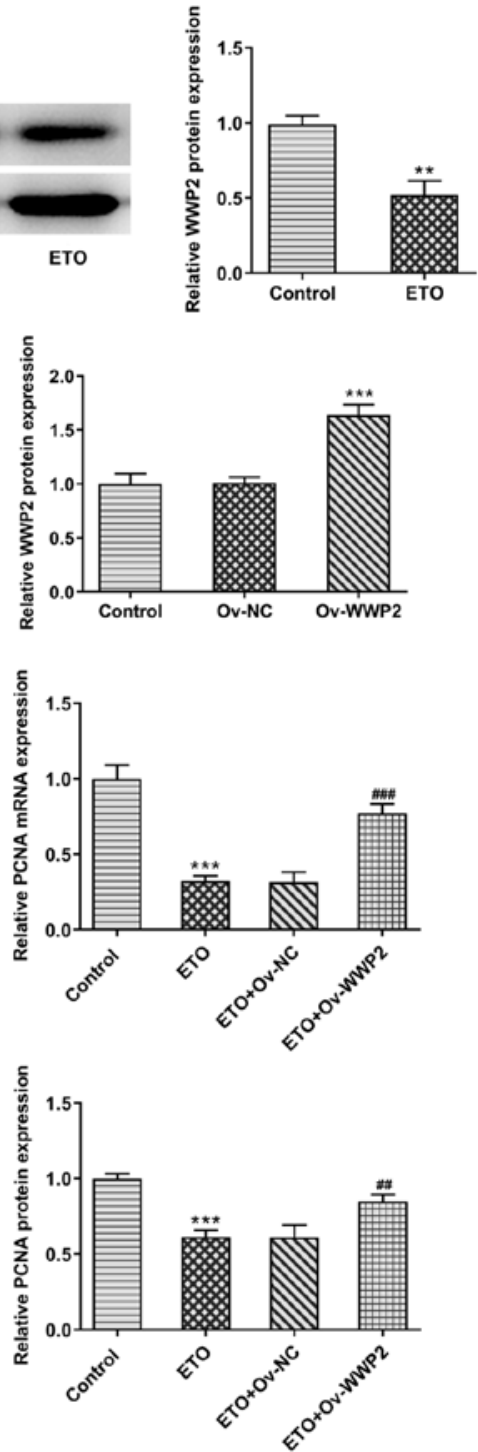

I

Control

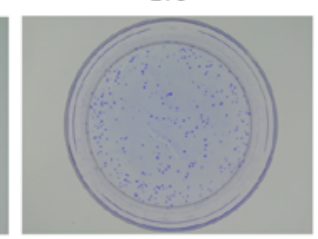

ETO+OV-WWP2
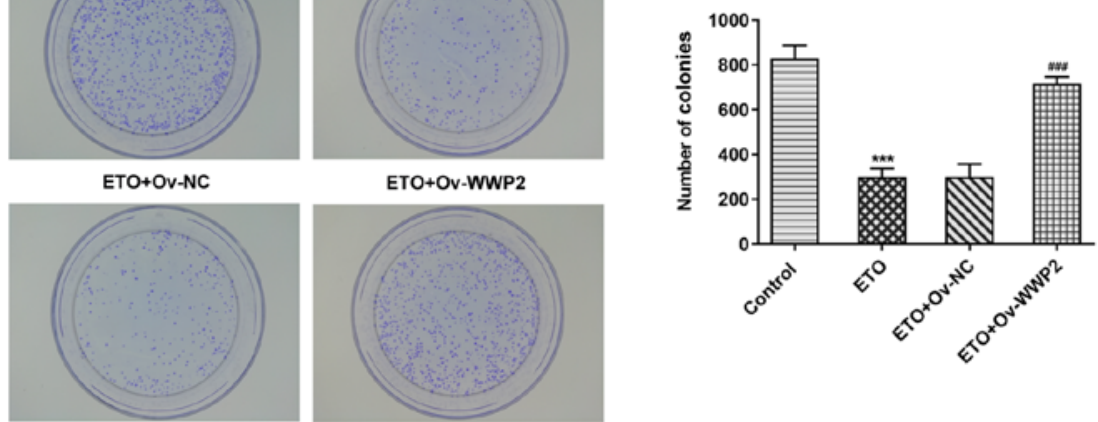

Figure 3. WWP2 overexpression abrogates the inhibitory effects of ETO on A549 cell proliferation. (A) The interaction between ETO and WWP2 was predicted using the STITCH database. A549 cells were treated with $3 \mu \mathrm{g} / \mathrm{ml}$ ETO for $24 \mathrm{~h}$, before the (B) mRNA and (C) protein expression levels of WWP2 were measured by RT-qPCR and western blot analyses, respectively. (D and E) A549 cells were transfected with pcDNA-WWP2 to overexpress WWP2. (D) The mRNA and (E) protein expression levels of WWP2 were measured by RT-qPCR and western blot analyses, respectively. (F-I) A549 cells overexpressing or not overexpressing WWP2 were treated with $3 \mu \mathrm{g} / \mathrm{ml}$ ETO for $24 \mathrm{~h}$. (F) Cell viability was assessed using the Cell Counting Kit- 8 assay. (G) mRNA and (H) protein levels of Ki67 and PCNA were determined by RT-qPCR and western blot assays, respectively. (I) Cell proliferation was assessed and quantified by colony formation assay. ${ }^{* *} \mathrm{P}<0.01$ and ${ }^{* * * *} \mathrm{P}<0.001$ vs. Control; ${ }^{\# \#} \mathrm{P}<0.01$ and ${ }^{\# \# \#} \mathrm{P}<0.001$ vs. ETO + ov-NC. WWP2, WW domain containing E3 ubiquitin protein ligase 2; ETO, etomidate; PCNA, proliferating cell nuclear antigen; ov-NC, overexpression with negative control vector; RT-qPCR, reverse transcription-quantitative PCR.

ETO negatively regulates the expression of WWP2 in A549 cells. Subsequently, the present study further investigated the mechanism underlying the effects of ETO on NSCLC. Bioinformatics analysis using the STITCH database predicted 

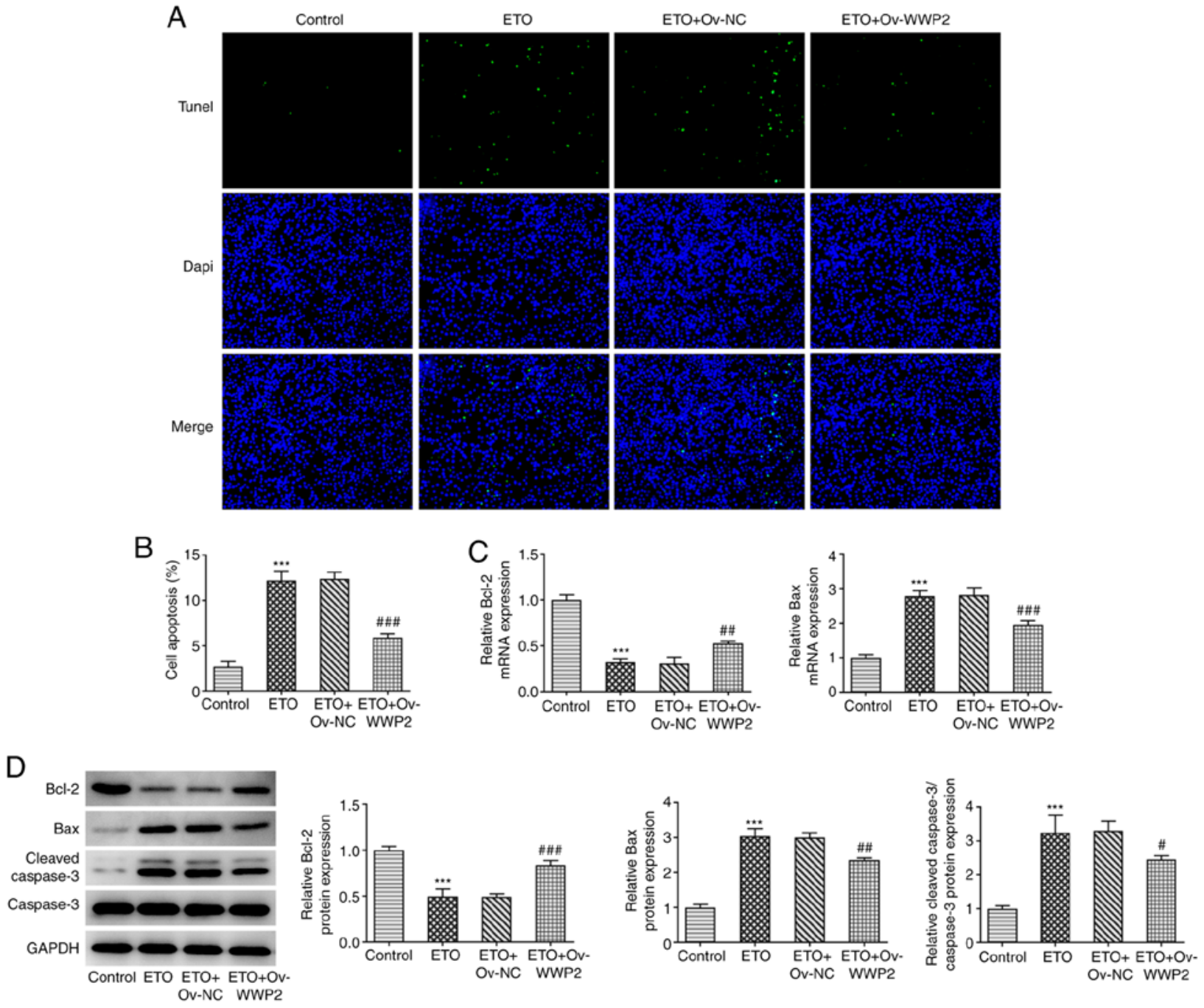

Figure 4. WWP2 overexpression abrogates the potentiating effects of ETO on A549 cell apoptosis. A549 cells overexpressing or not WWP2 were treated with $3 \mu \mathrm{g} / \mathrm{ml}$ ETO for $24 \mathrm{~h}$. (A) Cell apoptosis was evaluated by TUNEL assay (Magnification, $\mathrm{x} 200$ ), (B) which was quantified. (C) mRNA levels of Bcl-2 and Bax were detected by reverse transcription-quantitative PCR. (D) Protein expression levels of Bcl-2, Bax, cleaved caspase 3 and caspase 3 were determined by western blot analysis. ${ }^{* * *} \mathrm{P}<0.001$ vs. Control. ${ }^{\#} \mathrm{P}<0.05,{ }^{\# \#} \mathrm{P}<0.01$ and ${ }^{\# \# \#} \mathrm{P}<0.001$ vs. ETO + ov-NC. WWP2, WW domain containing E3 ubiquitin protein ligase 2; ETO, etomidate; ov-NC, overexpression with negative control vector.

that ETO could interact with WWP2 and PTEN by upregulating the protein expression of WWP2 and downregulating the protein expression of PTEN. (Fig. 3A). Data from RT-qPCR and western blot analyzes demonstrated that, compared with that in the control group, treatment of A549 cells with $3 \mu \mathrm{g} / \mathrm{ml}$ ETO significantly downregulated WWP2 expression (Fig. 3B and C).

ETO attenuates A549 cell proliferation and induces apoptosis via downregulating WWP2. A549 cells were then transected with the pcDNA3.1-WWP2 plasmid to overexpress WWP2 (ov-WWP2). As shown in Fig. 3D and E, the expression of WWP2 in the ov-WWP2 group was significantly increased compared with that in the cell group transfected with the empty plasmid (ov-NC). Additionally, WWP2 overexpression significantly reversed the inhibitory effects of ETO on A549 cell viability, colony formation, Ki67 and PCNA expression (Fig. 3F-I). Results from TUNEL assay revealed that WWP2 overexpression significantly reversed the potentiating effects of ETO on A549 cell apoptosis (Fig. 4A and B). In addition, the mRNA levels of Bcl-2 and Bax, in addition to the protein expression levels of Bcl-2, Bax, cleaved caspase 3 and caspase 3 were detected by RT-qPCR and western blotting. The results showed that compared with that in the ETO + OV-NC group, the expression levels of Bcl-2 protein and relative Bcl-2 mRNA in ETO + OV-WWP2 group were significantly increased, whilst the expression levels of Bax protein and relative Bax mRNA were downregulated, indicating that WWP2 overexpression significantly reversed the potentiating effects of ETO on A549 cell apoptosis. (Fig. 4C and D).

ETO attenuates the physiology of A549 cells by PTEN downregulation through targeting WWP2. Subsequently, the mRNA and protein expression levels of PTEN were evaluated by RT-qPCR and western blot analyzes, respectively. As shown in Fig. 5A and B, ETO significantly increased PTEN expression compared with that in the control group, which was significantly reversed by WWP2 overexpression. In addition, the significantly decreased AKT phosphorylation and PI3K 

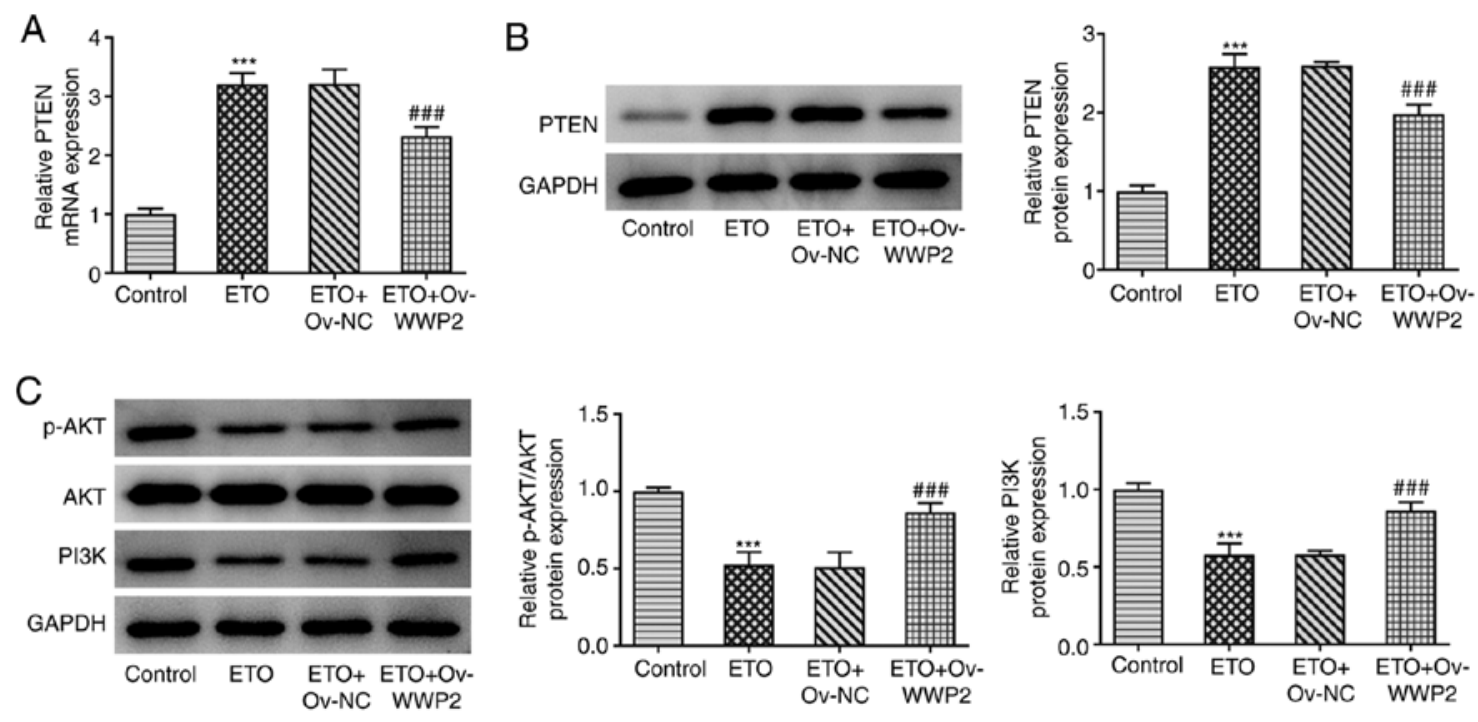

Figure 5. ETO upregulates PTEN and inhibits the activation of the PI3K/ATK pathway, which were reversed by WWP2 overexpression. A549 cells overexpressing or not overexpressing WWP2 were treated with $3 \mu \mathrm{g} / \mathrm{ml}$ ETO for $24 \mathrm{~h}$. The (A) mRNA and (B) protein expression levels of PTEN were determined by reverse transcription-quantitative PCR and western blot analysis, respectively. (C) Protein levels of p-AKT/AKT and PI3K were detected by western blot analysis. ${ }^{* * *} \mathrm{P}<0.001$ vs. Control. ${ }^{\# \#} \mathrm{P}<0.001$ vs. ETO + ov-NC. ETO, etomidate; WWP2, WW domain containing E3 ubiquitin protein ligase 2 ; ov-NC, overexpression with negative control vector.

expression induced by ETO were also in turn significantly reversed by WWP2 overexpression (Fig. 5C).

\section{Discussion}

Lung cancer is one of the most common malignancies worldwide, of which NSCLC is the most prevalent type of lung cancer, accounting for $\sim 80 \%$ of all lung cancer cases (20). Due to the lack of effective long-term treatment strategies and difficulties in early-stage diagnosis, the postoperative survival rate of patients with NSCLC remains low. Previous studies have shown that among patients with advanced NSCLC who have previously received operative, chemotherapy or radiotherapy treatment, the 5-year overall survival rate of all treated patients $(n=129)$ was estimated to be $16 \%(21,22)$. Therefore, identifying novel treatment approaches is crucial for improving the prognosis of patients with NSCLC. In the present study, the results demonstrated that ETO could attenuate proliferation whilst inducing apoptosis in A549 cells in a dose-dependent manner. In addition, the interaction between WWP2 and ETO was predicted using the STITCH database, where WWP2 overexpression was subsequently found to reverse the inhibitory effects of ETO on A549 cell activity.

ETO is a commonly used hypnotic and intravenous anesthetic (23). Previous studies have shown that ETO exerts antioxidant, anti-inflammatory, antitumor and antiplatelet aggregation effects $(24,25)$. For example, ETO could reduce the proliferation, migration and invasion of human adrenocortical cancer cells (9) and induce the apoptosis of N2a brain tumor cells (10). In lung cancer, one previous study demonstrated that ETO can effectively attenuate the proliferation and migration of A549 cells, supporting the notion of antitumor effects of ETO on NSCLC (11). However, the specific role and mechanism of action of ETO in NSCLC remain elusive. ETO treatment conferred no effects on the immune system of patients with lung cancer (26). Therefore, the effect of ETO on
NSCLC is worthy of further investigation. In the present study, ETO significantly attenuated the cell viability and proliferation of A549 cells, whilst promoting apoptosis in a dose-dependent manner. Therefore, the results of the present study further supported the potential antitumor and therapeutic value of ETO in NSCLC.

Furthermore, the present study further investigated the mechanism underlying the effect of ETO on NSCLC. Bioinformatics analysis by the STITCH database revealed that WWP2 could interact with ETO. WWP2 belongs to the ubiquitin ligase protein family and has been reported to serve an important role in liver cancer and lung adenocarcinoma (27). Previous studies in prostate cancer models have shown that WWP2 served as an oncogene, which mainly operated through the PTEN/Akt signaling pathway to promote carcinogenesis $(14,28)$, In gastric cancer, overexpression of WWP2 enhanced cell proliferation by silencing PTEN protein expression and upregulating of Akt phosphorylation (29). Loss of PTEN protein expression has been widely reported in several types of malignant tumors, including gastric cancer, liver cancer and lung adenocarcinoma, where they are closely associated with histological grade, metastasis and prognosis (30-32). PTEN lie upstream of the PI3K/AKT signaling pathway and functions as an important regulator of non-small cell lung cancer (33). A previous study showed that PTEN played an inhibitory role on Human cervical cancer cells (HeLa), human prostate cancer cells (DU145) and human prostatic hyperplasia cells (BPH1) by negatively regulating the PI3K/Akt signaling pathway (28). Downstream, the PI3K/AKT pathway regulates various cellular functions during tumorigenesis and development, including cell proliferation, migration and apoptosis, thereby serving a key role in promoting cancer progression (29). It has been suggested that ETO can reduce PI3K/AKT activation in A549 cells (11). Therefore, in the present study it was hypothesized that ETO may act through this pathway. It was found that PTEN and 
WWP2 could interact with each other. WWP2 was previously found to promote the proliferation of gastric cancer cells in a PTEN-dependent manner in gastric cancer (29). WWP2 was also found to be highly expressed in NSCLC, suggesting that it may function as a tumor-promoting factor (16). Therefore, the present study investigated the effects of WWP2 on the proliferation of NSCLC cells and the PTEN/PI3K/AKT axis. Treatment of A549 cells with ETO inhibited the PI3K/AKT signaling pathway by downregulating WWP2 and upregulating PTEN, which also attenuated A549 cell proliferation and enhancing apoptosis.

However, it should be noted that there are limitations in the present study. Only one cell line was used for present study. In future studies, multiple NSCLC cell lines must be used for in vitro experiments for more comprehensive and in-depth validation. A549 cells are also of the wild-type p53 genotype, whilst most other lung cancer cell lines contain a mutated p53 genotype. Since p53 is one of the key mediators of apoptosis (34), the role of ETO in cell lines with mutant p53 should be explored. In addition, ETO was not only found to interact with WWP2, but also with eight other proteins, namely cytochrome P450, family 11, subfamily B, polypeptide 2, cytochrome P450, family 11 , subfamily B, polypeptide $1, \gamma$-aminobutyric acid (GABA) A receptor $\alpha 1$, ADRA2B: adrenoceptor $\alpha 2 \mathrm{~B}$, sulfotransferase family, cytosolic, 2A, dehydroepiandrosterone-preferring, member 1, GABA A receptor $\gamma 2$, unc-13 homolog $B$ and GABA A receptor $\gamma 1$, which should be further explored in future studies. The molecular mechanism of ETO and WWP2/PTEN on NSCLC cell function has not been fully investigated in the present study. These issues require further in-depth analysis and should be addressed in future studies.

Overall, results of the present study demonstrated that ETO reduced the prolfieration of NSCLC cells in a dose-dependent manner. The mechanism underlying the effects of ETO on NSCLC may be associated with the downregulation of WWP2 and activation of PTEN. These findings may provide a theoretical basis for the clinical treatment of NSCLC using ETO.

\section{Acknowledgements}

Not applicable.

\section{Funding}

No funding was received.

\section{Availability of data and materials}

The datasets used and/or analyzed during the current study are available from the corresponding author on reasonable request.

\section{Authors' contributions}

$\mathrm{XM}$ and DL contributed to conception and design of the study. DL, JZ and LY contributed to the experiments and data collection. $\mathrm{ZJ}$ and $\mathrm{XC}$ contributed to analysis and interpretation of data. XM revised the manuscript critically for important intellectual content. XM and DL confirmed the authenticity of all the raw data. All authors read and approved the final version of the manuscript.

\section{Ethics approval and consent to participate}

Not applicable.

\section{Patient consent for publication}

Not applicable.

\section{Competing interests}

The authors declare that they have no competing interests.

\section{References}

1. Miller KD, Nogueira L, Mariotto AB, Rowland JH, Yabroff KR, Alfano CM, Jemal A, Kramer JL and Siegel RL: Cancer treatment and survivorship statistics, 2019. CA Cancer J Clin 69: 363-385, 2019.

2. Siegel RL, Miller KD and Jemal A: Cancer statistics, 2019. CA Cancer J Clin 69: 7-34, 2019.

3. Hoffman PC, Mauer AM and Vokes EE: Lung cancer. Lancet 355: 479-485, 2000.

4. Vinod SK: International patterns of radiotherapy practice for non-small cell lung cancer. Semin Radiat Oncol 25: 143-150, 2015.

5. Gandhi L, Rodríguez-Abreu D, Gadgeel S, Esteban E, Felip E, De Angelis F, Domine M, Clingan P, Hochmair MJ, Powell SF, et al: Pembrolizumab plus chemotherapy in metastatic non-small-cell lung cancer. N Engl J Med 378: 2078-2092, 2018.

6. Groth SS, Rueth NM, Hodges JS, Habermann EB, Andrade RS D'Cunha J and Maddaus MA: Conditional cancer-specific versus cardiovascular-specific survival after lobectomy for stage I non-small cell lung cancer. Ann Thorac Surg 90: 375-382, 2010

7. Herbst RS, Heymach JV and Lippman SM: Lung cancer. N Engl J Med 359: 1367-1380, 2008.

8. Erdoes G, Basciani RM and Eberle B: Etomidate-a review of robust evidence for its use in various clinical scenarios. Acta Anaesthesiol Scand 58: 380-389, 2014

9. Fassnacht M, Hahner S, Beuschlein F, Klink A, Reincke M and Allolio B: New mechanisms of adrenostatic compounds in a human adrenocortical cancer cell line. Eur J Clin Invest 30 (Suppl 3): S76-S82, 2000.

10. Chen HT, Zhou J, Fan YL, Lei CL, Li BJ, Fan LX, Xu L, Xu M, Hu XQ and Yu ZY: Anesthetic agent etiomidate induces apoptosis in N2a brain tumor cell line. Mol Med Rep 18: 3137-3142, 2018.

11. Chu CN, Wu KC, Chung WS, Zheng LC, Juan TK, Hsiao YT, Peng SF, Yang JL, Ma YS, Wu RS and Chung JG: Etomidate suppresses invasion and migration of human A549 lung adenocarcinoma cells. Anticancer Res 39: 215-223, 2019.

12. Bernassola F, Karin M, Ciechanover A and Melino G: The HECT family of E3 ubiquitin ligases: Multiple players in cancer development. Cancer Cell 14: 10-21, 2008.

13. Mokuda S, Nakamichi R, Matsuzaki T, Ito Y, Sato T, Miyata K, Inui M, Olmer M, Sugiyama E, Lotz $\mathrm{M}$ and Asahara $\mathrm{H}$ : Wwp2 maintains cartilage homeostasis through regulation of Adamts5. Nat Commun 10: 2429, 2019.

14. Zhang R, Zhang J, Luo W, Luo Z and Shi S: WWP2 is one promising novel oncogene. Pathol Oncol Res 25: 443-446, 2019.

15. Ding ZY, Huang YJ, Tang JD, Li G, Jiang PQ and Wu HT: Silencing of hypoxia-inducible factor-1 $\alpha$ promotes thyroid cancer cell apoptosis and inhibits invasion by downregulating WWP2, WWP9, VEGF and VEGFR2. Exp Ther Med 12: 3735-3741, 2016.

16. Yang R, He Y, Chen S, Lu X, Huang C and Zhang G: Elevated expression of WWP2 in human lung adenocarcinoma and its effect on migration and invasion. Biochem Biophys Res Commun 479: 146-151, 2016. 
17. Kuhn M, von Mering C, Campillos M, Jensen LJ and Bork P STITCH: Interaction networks of chemicals and proteins. Nucleic Acids Res 36 (Database Issue): D684-D688, 2008.

18. Livak KJ and Schmittgen TD: Analysis of relative gene expression data using real-time quantitative PCR and the 2(-Delta Delta C(T)) method. Methods 25: 402-408, 2001.

19. Liao XH,Lu DL, Wang N,Liu LY, Wang Y,Li YQ, Yan TB, Sun XG, $\mathrm{Hu} \mathrm{P}$ and Zhang TC: Estrogen receptor $\alpha$ mediates proliferation of breast cancer MCF-7 cells via a p21/PCNA/E2F1-dependent pathway. FEBS J 281: 927-942, 2014.

20. Dey N, De P and Leyland-Jones B: PI3K-AKT-mTOR inhibitors in breast cancers: From tumor cell signaling to clinical trials. Pharmacol Ther 175: 91-106, 2017.

21. Gettinger S, Horn L, Jackman D, Spigel D, Antonia S, Hellmann M, Powderly J, Heist R, Sequist LV, Smith DC, et al: Five-year follow-up of nivolumab in previously treated advanced non-small-cell lung cancer: Results from the CA209-003 study. J Clin Oncol 36: 1675-1684, 2018.

22. Garon EB, Hellmann MD, Rizvi NA, Carcereny E, Leighl NB, Ahn MJ, Eder JP, Balmanoukian AS, Aggarwal C, Horn L, et al: Five-year overall survival for patients with advanced non-small-cell lung cancer treated with pembrolizumab: Results from the phase I KEYNOTE-001 study. J Clin Oncol 37: 2518-2527, 2019

23. McGrath M, Ma C and Raines DE: Dimethoxy-etomidate: A nonhypnotic etomidate analog that potently inhibits steroidogenesis. J Pharmacol Exp Ther 364: 229-237, 2018.

24. Forman SA: Clinical and molecular pharmacology of etomidate. Anesthesiology 114: 695-707, 2011.

25. Av Sá LGD, Silva CRD, de A Neto JB, Cândido TM, de Oliveira LC, do Nascimento FB, Barroso FD, da Silva LJ, de Mesquita JR, de Moraes MO, et al: Etomidate inhibits the growth of MRSA and exhibits synergism with oxacillin. Future Microbiol 15: 1611-1619, 2020.

26. Liu J, Dong W, Wang T, Liu L, Zhan L, Shi Y and Han J: Effects of etomidate and propofol on immune function in patients with lung adenocarcinoma. Am J Transl Res 8: 5748-5755, 2016.
27. Chantry A: WWP2 ubiquitin ligase and its isoforms: New biological insight and promising disease targets. Cell Cycle 10 2437-2439, 2011

28. Maddika S, Kavela S, Rani N, Palicharla VR, Pokorny JL, Sarkaria JN and Chen J: WWP2 is an E3 ubiquitin ligase for PTEN. Nat Cell Biol 13: 728-733, 2011.

29. Wang K, Liu J, Zhao X, Li H, Luo G, Yu Y, Guo Y, Zhang L, Zhu J, Wang S, et al: WWP2 regulates proliferation of gastric cancer cells in a PTEN-dependent manner. Biochem Biophys Res Commun 521: 652-659, 2020.

30. Dillon LM and Miller TW: Therapeutic targeting of cancers with loss of PTEN function. Curr Drug Targets 15: 65-79, 2014.

31. Trigka EA, Levidou G, Saetta AA, Chatziandreou I, Tomos P, Thalassinos N, Anastasiou N, Spartalis E, Kavantzas N, Patsouris E and Korkolopoulou P: A detailed immunohistochemical analysis of the PI3K/AKT/mTOR pathway in lung cancer: Correlation with PIK3CA, AKT1, K-RAS or PTEN mutational status and clinicopathological features. Oncol Rep 30: 623-636, 2013.

32. Daniilidou K, Frangou-Plemenou M, Grammatikakis J, Grigoriou O, Vitoratos N and Kondi-Pafiti A: Prognostic significance and diagnostic value of PTEN and p53 expression in endometrial carcinoma. A retrospective clinicopathological and immunohistochemical study. J BUON 18: 195-201, 2013.

33. Pérez-Ramírez C, Cañadas-Garre M, Molina MÁ, Faus-Dáder MJ and Calleja-Hernández MÁ:PTEN and PI3K/AKT in non-small-cell lung cancer. Pharmacogenomics 16: 1843-1862, 2015.

34. Soond SM, Savvateeva LV, Makarov VA, Gorokhovets NV, Townsend PA and Zamyatnin AA Jr: Making connections: p53 and the cathepsin proteases as co-regulators of cancer and apoptosis. Cancers (Basel) 12: 3476, 2020.

This work is licensed under a Creative Commons Attribution-NonCommercial-NoDerivatives 4.0 International (CC BY-NC-ND 4.0) License. 